\title{
Marketing gefragt
}

\author{
Ludger Kolhoff
}

Das Persönliche Budget macht Menschen mit Behinderung zu Kunden. Die Leistungsanbieter müssen sich deshalb mehr Gedanken um die Vermarktung ihrer Angebote machen. Und sie müssen ihre Mitarbeiterinnen und Mitarbeiter auf mögliche Zwickmühlen im professionellen Handeln vorbereiten.

In der Vergangenheit galt in der Behindertenhilfe das Sachleistungsprinzip: Die Leistungen wurden von Einrichtungen erbracht und dem Leistungsberechtigten zur Verfügung gestellt. Die Einrichtungen erhielten hierfür Geldleistungen von den Kostenträgern. Es gab ein Dreiecksverhältnis der Leistungserbringung zwischen Leistungserbringer (Einrichtung), Leistungsempfänger (behinderte Person) und Kostenträger (z. B. Träger der Sozialhilfe). Dieses Modell der Leistungserbringung wurde durch die Einführung des Persönlichen Budgets verändert.

Seit Januar 2008 können Menschen mit Behinderung über einen Geldbetrag zur Teilhabe am Leben in der Gemeinschaft anstelle von Sachleistungen verfügen. Durch die Einführung dieses Persönlichen Budgets soll die Teilhabe und Selbstbestimmung von Menschen mit Behinderung gestärkt werden. Menschen mit Behinderung sollen in die Lage versetzt werden, Angebote zu nutzen, die ihren individuellen Bedürfnissen entsprechen. Wenn sich Menschen mit Behinderung statt für die Sachleistung für das Persönliche Budget entscheiden, werden sie zu Käufern, Kunden und manchmal zu Arbeitgebern, das heißt, die Leistungserbringer stehen in keinem Vertragsverhältnis mehr zum Kostenträger, sondern nur noch zum Leistungsempfänger. Für den Teilbereich der Behindertenhilfe, der durch das Persönliche Budget gesteuert wird, bedeutet dies, dass Marktmechanismen greifen. Die Kostenträger erwarten von diesen Marktmechanismen Einsparungen und einen Beitrag zur Lösung ihrer Finanzierungsprobleme. Es ist davon auszugehen, dass es mittelfristig zu Veränderungen in den Einrichtungen der Behindertenhilfe kommen wird. Da der Mensch mit Behinderung selbst entscheiden kann, was er für Leistungen haben möchte und wer sie wie, wann und wie oft erbringen soll, bekommt er eine ganz andere Position als vorher. Er kann Leistungen einkaufen oder gar Personal anstellen und entlassen.

Einrichtungen der Behindertenhilfe müssen sich dem stellen und sich mit dem Nachfrageverhalten, also den Wünschen ihrer Kunden auseinandersetzen, wenn sie auf dem neu entstehenden Käufermarkt bestehen wollen.

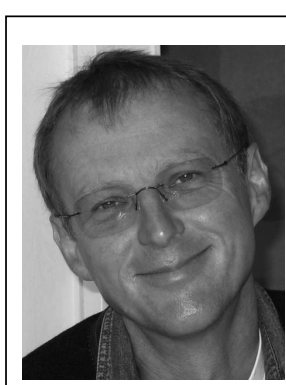

Prof. Dr. phil. MA Ludger Kolhoff (51) ist seit 1993 Professor für »Soziales Management « am Fachbereich Sozialwesen der Fachhochschule Braunschweig/ Wolfenbüttel mit den Schwerpunkten: Finanzierung, Personalwesen, Existenzgründung, Organisation und Organisationsentwicklung in sozialen Einrichtungen. Er leitet den ersten in Deutschland akkreditierten und reakkreditierten Studiengang zum »Master of Social Management «.

Internet http://www.fh-wolfenbuettel.de/cms/de/ fbs/not_in_menu/Kolhoff/personenkolhoff

Erfahrungen aus Nachbarländern, in denen das Persönliche Budget schon eingeführt wurde, zeigen, dass es zu einer Deinstitutionalisierung kam. So hat sich beispielsweise die Angebotsstruktur in den Niederlanden extrem verändert. Zunehmend schließen stationäre Einrichtungen zugunsten ambulanter Angebote und es werden neue Leistungsmodule entwickelt (Wacker/Wannsing/Schäfers $2005,47)$. Ähnliches ist aus Großbritannien und Schweden zu vermelden. Auch hier werden zunehmend stationäre Großeinrichtungen geschlossen und differenzierte und flexible Leistungsangebote entwickelt. Ob dies auch in Deutschland der Fall sein wird, muss sich noch zeigen. Erste Erfahrungen, beispielsweise aus Rheinland-Pfalz, bestätigen diese Entwicklung für Deutschland noch nicht, doch gehen Fachleute davon aus, dass ähnlich wie in den Niederlanden der Zuspruch zunehmen wird. In den Niederlanden nahmen Ende 2001 rund 36.000 Menschen das Persönliche Budget in Anspruch. Am Jahresende 2004 waren es bereits 70.000 Menschen (Wacker/Wannsing/Schäfers 2005, 47). Hier lagen die Tarife 25 Prozent unter den Tarifen für entsprechende Sachleistungen. Die Kostenträger gehen davon aus, dass ein ähnlicher Trend auch in Deutschland einsetzen wird und somit der starke $\mathrm{Zu}$ wachs der Ausgaben für den Behindertenbereich abgebremst werden kann.

Das Persönliche Budget soll die Kosten aller bisher individuell festgestellten Leistungen nicht überschreiten. Diesen individuellen Bedarf gilt es in einem ersten Schritt festzustellen. Hierzu werden Hilfeplan-Konferenzen durchgeführt, in denen der Betreuungsbedarf des Antragstellers als Stundenzahl durch den Sozialhilfeträger und nach Vorschlägen eines Leistungsanbieters festgelegt 
wird. Die Leistungsspanne umfasst den Umgang mit der eigenen Person, Basis- und Selbstversorgung, Alltagsleben, Tagesgestaltung, Freizeit, Arbeit, Beschäftigung, Ausbildung, Schule, soziale und administrative Angelegenheiten. Die Ermittlung individueller Unterstützungsmerkmale kann so erfolgen:

- eine Analyse verfügbarer und wahrgenommener Ressourcen

- eine Einschätzung erforderlicher Leistungen

- die Bildung von Budgets.

Es werden also Bedarfe eruiert und soziale Ressourcen (Freunde, Verwandte, Nachbarn) und persönliche Aktivitäten erfasst und einzelnen Teilbereichen (Haushaltsführung, Mobilität, soziale Beziehungen, Gesundheit, Kommunikation, Umgang mit Geld, Bildung, Arbeit, Freizeit, Erholung etc.) zugeordnet. In einem zweiten Schritt werden dann die erforderlichen Leistungen abgeleitet, beispielsweise Assistenz, Kompetenzförderung, Beratung und es wird das Ausmaß der Leistungen festgelegt. Dies ist die Grundlage der Budgetbestimmung. Die Erfahrungen aus bisherigen Modellversuchen zeigten, dass das Persönliche Budget zwischen 36 Euro und 12.683 Euro betrug. Die Mehrheit der bewilligten Budgetsummen lag zwischen 200 und 800 Euro (Deutscher Bundestag, Drucksache $16 / 3983,49)$.

Ein Kernbestandteil des Instrumentariums ist die Zielbestimmung. Die Ziele sollen gemäß der Smart-Formel sein: spezifisch, messbar, anspruchsvoll, realistisch und terminiert. Spezifisch sind sie dann, wenn sie einzelfallbezogen sind, messbar, wenn es qualitative und quantitative Indikatoren zur Überprüfung gibt; anspruchsvoll sind sie, wenn die Ziele fördernde und fordernde Entwicklungen dertenhilfe Schritt für Schritt verändern wird. Denn durch die Tatsache, dass Menschen mit Behinderung Wahlmöglichkeiten zur Verfügung gestellt werden und sie zwischen Sach- und Geldleistung wählen, beziehungsweise gleichzeitig Sachleistungen und ein Persönliches Budget für verschiedene Hilfen erhalten können, wird sich bei der Hilfebedarfsplanung ein Wandel von der Einrichtungsperspektive zur Personenperspektive ergeben. Die Anbieter von Leistungen zur Teilhabe müssen sich verstärkt mit dem Nachfrageverhalten der Menschen mit Behinderung auseinandersetzen.

Auch die Leistungen selbst werden sich meiner Meinung nach verändern: Komplexe Leistungen werden zugunsten flexibel kombinierbarer Leistungspakete aufgelöst. Es wird sich ein Trend zur angepassten Hilfe herausbilden, es geht also nicht mehr darum, behinderte Menschen mit fertigen Hilfemodellen zu versorgen, sondern sie zu Ko-Produzenten ihrer eigenen Hilfeplanung werden zu lassen.

Da die Einführung des Persönlichen Budgets immer mehr Einrichtungen der Behindertenhilfe vor die Herausforderung stellt, in einem Käufermarkt bestehen zu müssen, gilt es das Angebot auszudifferenzieren und die Marketinginstrumente der Leistungs-, Preis-, Distributionsund Kommunikationspolitik zu nutzen.

\section{Leistungspolitik}

Bei der Leistungspolitik geht es um die Gestaltung des konkreten Angebots auf der Grundlage einer Analyse des vorhandenen Angebots und der Erwartungen der Menschen mit Behinderung. Folglich gilt es zu klären, welche Leistungen die Organisationen anbieten, welche Veränderungen auf dem Hintergrund des Persönlichen Budgets zu erwarten sind, welche Erwartungen Menschen mit Behinderung haben, zu überprü-

beinhalten, realistisch, wenn sie im Rahmen der persönlichen und objektiven Rahmenbedingungen erreichbar sind und terminiert, wenn es feste Zeiträume zur Zielüberprüfung gibt.

Der Nachweis über die Verwendung des Persönlichen Budgets erfolgt auf der Grundlage dieser Zielvereinbarungen, und eine Kontrolle sollte im Sinne einer Ergebnisqualitätskontrolle in möglichst einfacher und unbürokratischer Form erfolgen, um die Eigenverantwortung und Selbstbestimmung des Budgetnehmers zu stärken. Die Nachweiskontrolle ist abhängig von der Art und dem Bedarf der Leistungen.

\section{Auswirkungen auf die Behindertenhilfe}

Es ist davon auszugehen, dass sich durch die Einführung des Persönlichen Budgets der Bereich der Behin- fen, ob veraltete, überflüssige, nicht genügend nachgefragte Leistungen existieren, ob Leistungen verbessert und kostengünstiger angeboten werden können, ob gegebenenfalls Leistungen gestrichen und neue, zusätzliche Leistungen angeboten werden sollen, ob der Leistungsmix insgesamt sinnvoll ist und ob Auslagerungen, Kooperationen oder Vernetzungen möglich sind.

\section{Preispolitik}

In jedem Käufermarkt, auch in dem der Behindertenhilfe, der durch die Einführung des Persönlichen Budgets geschaffen wird, spielt der Preis eine entscheidende Rolle. Um in einem sich entwickelnden Markt bestehen zu können, gilt es nicht nur die Konkurrenz und die Kosten im Blick zu haben, sondern sich insbesondere auch mit dem Kundennutzen auseinanderzusetzen. Denn je höher der Nutzen für einen behinderten Menschen ist, desto eher 
wird er bereit sein, einen Teil seines Budgets dafür zu verwenden.

Nun stellt sich die Frage, ob für den Menschen mit Behinderung die Kosten im Mittelpunkt stehen oder ob der persönliche Kontakt zur Einrichtung und zu den mit den Menschen arbeitenden Einrichtungen nicht noch wichtiger ist. Stehen die Kosten im Mittelpunkt, so gilt es hier anzusetzen, preiswerte Angebote vorzuhalten und neue Finanzierungsquellen zu eruieren. Neben dem Persönlichen Budget gilt es Zuschüsse und Zuwendungen, aber auch Sponsoring und Spendeneinnahmen zu akquirieren. Gerade für Behinderteneinrichtungen bietet sich hier ein weites Feld. Die Behindertenhilfe ist ein »Produkt «, das sich gut für das Fundraising eignet, aber beispielsweise auch für die Aktivierung ehrenamtlicher Mitarbeiter.

\section{Distributionspolitik}

In diesem Kontext gilt es auch zu eruieren, ob es Änderungsbedarf bei der Distributionspolitik gibt. Distribution umfasst die Gestaltung der Absatzwege, der logistischen Systeme und der Orte der Dienstleistungserstellung. Es stellt sich die Frage, wo der Austausch der Dienstleistung stattfindet, wie der Standort bewertet wird, ob man beispielsweise durch eine räumliche Verlegung von Verwaltungseinrichtungen den Austausch verbessern kann oder ob Zentralisierungen besser sind, ob Änderungen bei der zeitlichen Distribution der Leistungen sinnvoll sind. Zentrale Bedeutung für die Distributionspolitik haben die Absatzmittler. In diesem Kontext gilt es beispielsweise Kontakte zu Berufsbetreuern oder den Integrationsämtern zu pflegen und zu stärken.

\section{Kommunikationspolitik}

In einem sich entwickelnden Käufermarkt wird der Austausch durch Kommunikationsmittel geprägt. Es stellt sich die Frage, inwieweit mit Mitteln der Kommunikationspolitik Menschen mit Behinderung angesprochen werden können. In diesem Kontext gilt es zu klären, welche Medien genutzt, mit welchen Medien Menschen mit Behinderung angesprochen werden können. Hier sind die klassischen Marketinginstrumente nur bedingt einsetzbar. Viel wichtiger als Medien ist der direkte Kontakt mit Menschen mit Behinderung. Es geht darum, auf die Menschen mit Behinderung zuzugehen, Kontakt herzustellen, sicherzustellen, dass das Unterstützungsangebot, die Botschaft ankommt, dass die Botschaft positiv besetzt ist und das Leistungsangebot bei Menschen mit Behinderung verankert wird.

In diesem Kontext gilt es zu klären, welche Kommunikationsmittel schon eingesetzt werden, welche Medien besonders wichtig sind, ob es Hinweise auf die Wirksamkeit der bisherigen Maßnahmen gibt und welche weiteren Kommunikationsmöglichkeiten genutzt werden können.

$\mathrm{Zu}$ beachten sind Fragen des Involvements, der inneren Beteiligung, mit der die Kommunikation erfolgt. Weist der Mensch mit Behinderung ein hohes Involvement auf, wendet er also viel Zeit und Energie auf, um Informationen zu suchen oder ist seine Motivation, Informationen zu verarbeiten, eher gering? Einrichtungen, die sich neu auf dem Markt positionieren wollen, werden im Sinne eines Relationship-Managements Beziehungen zu den Kunden aufbauen und pflegen. Dieses Beziehungsmarketing wird insbesondere im Behindertenbereich stark von der Glaubwürdigkeit des Senders determiniert und ist nur erfolgreich, wenn Vertrauen geschaffen wird.

\section{Resümee}

Die Änderungen im Behindertenbereich sind als zweischneidiges Schwert zu sehen. So ist das Persönliche Budget einerseits ein partizipatives Instrument, dient aber auch andererseits dazu, Geld zu sparen. Es stellt sich die Frage, welche Konsequenzen dies für die Mitarbeitenden in der Behindertenhilfe hat, denn einerseits lautet ihr Auftrag: Integriere Menschen mit Behinderung, sorge beispielsweise dafür, dass sie auch am Arbeitsleben teilnehmen können. Doch wie soll das geschehen in einer Gesellschaft mit über drei Millionen Arbeitslosen? Und andererseits wird gefordert: Gebe weniger Geld aus, spare, konkurriere, sei preiswert und qualitativ besser als die Konkurrenz. In diesem Kontext besteht die Gefahr, dass die Mitarbeiter der Behindertenhilfe in Doublebind-Situationen geraten im Sinne eines »Du bist gut, aber schlecht «: Je nach Perspektive lautet die Botschaft entweder: Du arbeitest zwar mit Menschen mit Behinderung, willst aber damit Geld verdienen. Oder aus ökonomische Perspektive: Einige deiner Projekte und Betreuungen waren wirtschaftlich erfolgreich. Du hast Dich aber auch um Probleme und Menschen gekümmert, an denen Du nichts verdient hast. Die Botschaft lautet also: Das hast du gut gemacht, du schlechter Mensch oder aber: Das hast du schlecht gemacht, du guter Mensch.

Mitarbeitende und Studierende, die sich auf eine Arbeit in der Behindertenhilfe vorbereiten, müssen sich dieser Situation stellen und neue Kompetenzen erwerben, um mit diesem Doublebind umgehen zu können.

\section{Literatur}

Deutscher Bundestag 2006, 16 Wahlperiode, Drucksache 16/3983, Bericht der Bundesregierung über die Ausführungen der Leistungen des Persönlichen Budgets nach $\S 17$ des Neunten Buches Sozialgesetzbuch.

Wacker, E.; Wansing, G.; Schäfer, M.: Personenbezogene Unterstützung und Lebensqualität. Teilhabe mit einem Persönlichen Budget. Wiesbaden 2005. 